\title{
Grafted Surface Sheared by Short Polymers and the Total Entanglement Threshold
}

\author{
C. Gay $\left(^{*}\right)$
}

Laboratoire de Physique de la Matière Condensée $\left({ }^{* *}\right)$, Collège de France, 11 Place Marcelin Berthelot, 75231 Paris Cedex 05, France

(Received 18 September 1995, received in final form 7 December 1995, accepted 14 December 1995)

PACS.46.30.Pa - Friction, wear, adherence, hardness, mechanical contacts, and tribology

\begin{abstract}
The shear flow of a polymer melt ( $P$ monomers per chain) above a solid surface on which longer, chemically identical chains have been grafted $(N$ monomers, $N>P$ ), shows that friction at the surface is enhanced and that slippage is much reduced (with respect to the bare surface behavior), provided that the melt is entangled $\left(P>N_{\mathrm{e}}\right)$. One important issue in this problem is the drag number $X$ of the tethered chains, i.e. the number of melt chains that they trap. Recently, two different regimes were described $[1]$ for the opposite case $N<P$ : a Stokes regime with $X=N^{1 / 2}$ (total entanglement) and a Rouse regime with $X=N / N_{\mathrm{e}}$. Here, the structure of the drag number for $N>P$ is discussed: in this case, it is always linear in $N$, but long chains do display a coil-stretch transition. The value of the minimum molecular weight $M^{*}$ for this effect to take place is also discussed; star polymers should display the high molecular weight behavior even if they are smaller in size: this should allow for a test of the model.
\end{abstract}

Résumé. - Le cisaillement d'un polymère fondu ( $P$ monomères par molécule) au-dessus d'une surface solide greffée de chaînes chimiquement identiques et plus longues ( $N$ monomères, $N>P$ ). donne lieu à une friction plus élevée et à un glissement réduit par rapport au cas d'une surface lisse, dès lors que le fondu est enchevêtré $\left(P>N_{\mathrm{e}}\right)$. Le nombre $X$ de chaînes captées, c'est-àdire le nombre de molécules du fondu enchevêtrées avec les chaines greffées, est un paramètre important de la modélisation du problème. Deux différents régimes ont été récemment proposés [1] pour l'autre cas $N<P: X=N^{1 / 2}$ dans le régime dit de Stokes (enchevêtrement total), et un régime de Rouse avec $X=N / N_{\mathrm{e}}$. On discute ici de la valeur de $X$ lorsque $N>P: X$ est alors toujours proportionnel à $N$, mais une transition d'étirement est cependant prévue pour les longues chaînes. On discute aussi de la valeur du poids moléculaire critique au-delà duquel cet effet intervient; le comportement de polymères branchés, en étoile par exemple, devrait inclure cet effet même si leur taille est moindre: ceci pourrait constituer un test du modèle.

\section{Introduction}

It is known that polymer melts subjected to a shear flow slip on smooth, passive surfaces as a result of the high viscosity of the polymer melt relative to that of a liquid of monomers [2].

$\left({ }^{*}\right)$ e-mail: gay@ext.jussieu.fr

$\left({ }^{* *}\right)$ U.R.A. 792 du CNRS

(C) Les Éditions de Physique 1996 
Slippage gives rise to a finite velocity $V$ at the surface and can be characterized by the extrapolation length $b$, defined as the distance beyond the wall at which the velocity profile extrapolates to zero. In highly entangled melts this distance can reach a few microns.

If the solid surface has been roughened slippage is reduced; one very efficient way of doing this is to attach polymer chains to the surface. Due to entanglements between the grafted chains and the melt chains, friction is enhanced near the surface and slippage is very much reduced [3]; the extrapolation length $b$ can become as low as a coil size [4].

For a melt of sufficiently long chains $\left(P>N_{\mathrm{e}}\right)$, the classical picture [5,6] yields, regardless of the chain length, an average of one entanglement every $N_{\mathrm{e}}$ th monomer along any particular chain (an entanglement being some sort of topological constraint due to the presence of other chains). $N_{\mathrm{e}}$ is called the entanglement number. It can be estimated through various experimental methods (which lead to values that differ at most by a factor of order 3 or 4) [6]. Each chain is considered to be confined in a tube due to the constraints exerted by the surrounding chains. This Edward's tube has a diameter of order $D^{*}=a N_{\mathrm{e}}^{1 / 2}$, where $a$ is the monomer size, and $D^{*}$ is the average spatial distance between two consecutive entanglements along a particular chain.

In the slippage problem, friction at the surface is believed to arise through the following mechanism $[1,7]$ : suppose a mobile chain $P$ moves at velocity $V$ with respect to a grafted chain $N$ with which it happens to be entangled; then before it has moved an entanglement distance $D^{*}$, the $P$ chain has to slide over a fraction of its own tube length $L_{\mathrm{t}}=\left(P / N_{\mathrm{e}}\right) D^{*}$ in order to disentangle from the grafted chain [8]. The velocity of the $P$ chain along its tube is thus $V_{\mathrm{t}}=\left(P / N_{\mathrm{e}}\right) V$. The dissipation due to this enhanced sliding velocity is given by $T \dot{S} \equiv P \zeta_{1} V_{\mathrm{t}}^{2}$, where $\zeta_{1}$ is the coefficient of friction of a monomer in the surrounding liquid. If $f_{V}$ denotes the force acting on the grafted chain, due to the sliding motion of one melt chain, then the useful work dissipated at the interface is $f_{V} V$ per sliding chain. If we assume that this is a significant fraction of the total dissipation $T \dot{S}$ then the force $f_{V}$ is given by:

$$
f_{V}=P \zeta_{1}\left(\frac{P}{N_{\mathrm{e}}}\right)^{2} V=a \eta_{P} V,
$$

where $\eta_{P}$ is the melt viscosity. The grafted chain $N$ thus undergoes a force $f_{V}$ from each melt chain with which it is entangled.

Different approaches have been suggested to calculate the number $X$ of chains trapped by one $N$ chain, which is called the drag number. The first models $[3,7,9]$ suggested that $X$ was proportional to $N^{1 / 2}$. According to the binary entanglement model [1], however, $X$ is proportional to $N$ for moderate values of $N\left(N<N_{e}^{2}\right)$ : we call this the Rouse regime; on the other hand, $X$ is predicted to be proportional to $N^{1 / 2}$ for higher values $\left(N>N_{\mathrm{e}}^{2}\right)$ : this is the Stokes regime (the reason for this name is that the friction due to the tethered chain in this regime, is $F_{V}=N^{1 / 2} f_{V}=R \eta_{P} V$, where $R$ is the radius of gyration of the chain, and this shows that the chain behaves like a solid sphere with zero-velocity boundary conditions in a liquid of viscosity $\eta_{P}$ ). The early models with $X \propto N^{1 / 2}$ predict a coil-stretch transition of the grafted chains; this should lead to a non-monotonous plot of the extrapolation length $b(V)$ (like that of Fig. 3a). However, recent experiments on such systems [10] allow for a combined measure of slippage velocity and shear rate (and hence of the extrapolation length): no decrease of $b(V)$ seems to have been observed yet [11]; at present, it seems that only the results derived from the binary entanglement model (Refs. $[1,4]$ and the present paper) are compatible with this feature (see Fig. 3b).

Let us recall briefly the ideas of the binary entanglement model [1]: an entanglement is now supposed to be some topological constraint on the test chain, due to only one given, other chain 
(there is no such crude hypothesis in the classical picture). Let $R=a N^{1 / 2}$ denote the size of a chain. If we compare the number $n\left(R^{3}\right)=\left(a N^{1 / 2}\right)^{3} / N a^{3}=N a^{3}=N^{1 / 2}$ of chains present in the vicinity of the test chain and the available number $N / N_{\mathrm{e}}$ of entanglements along it, we are led to the following conclusion: for $X>N_{\mathrm{e}}^{2}$, the number of available entanglements is the higher; thus all chains are trapped $\left(X=N^{1 / 2}\right)$ and each is entangled several times with the test chain (this is the total entanglement, or Stokes regime). If, on the other hand, $N<N_{\mathrm{e}}^{2}$, then only a fraction of the surrounding chains is trapped ( $X=N / N_{\mathrm{e}}$, Rouse regime).

If on average $X$ melt chains are entangled with one tethered chain, the friction force acting onto the latter is given by:

$$
F_{V}=X f_{V}
$$

With the grafted surface ( $\nu$ tethered chains per unit area), we have to consider [3] that the shear stress $\sigma$ is transmitted to the surface both by mere friction of the melt molecules on the bare solid surface and through the entanglement friction force $F_{V}$ per grafted chain:

$$
\sigma=\frac{1}{a^{2}} \zeta_{1} V+\nu F_{V}
$$

(In practice, entanglement effects dominate most of the time and the first term is often negligible).

In the present paper we are concerned with the slippage of a melt of shorter chains $(P<N)$ and we must first estimate the drag number $X$ for this case (Sect. 2). As in the opposite case $P>N$ [4], we find a cooperative effect at high grafting densities. All these results coincide with those of reference [4] for the particular case $P=N$.

In Section 3, we discuss which molecular weight $M^{*}$ should correspond, for a real polymer, to the crossover between the Rouse and Stokes regimes described above. We also look at a system that should display the Stokes behavior $\left(X \propto N^{1 / 2}\right)$ although with smaller objects, namely grafted star-shaped polymers. This should allow for a test of the binary entanglement model.

\section{Slippage on a Grafted Surface: the $\mathbf{P}<\mathbf{N}$ Case}

As explained in the introduction, the crucial point for the description of slippage is the drag number $X$ of the tethered chains, i.e. the number of melt molecules that are trapped by each of the grafted chains. First we compute the drag number of a tethered chain as a function of its elongation; then we work out therefrom the behavior of a grafted surface.

2.1. Drag Number of a Tethered Chain in a Shorter Melt. - When a chain of chemical length $N$ is immersed in a melt of shorter, chemically identical chains ( $P$ monomers, $P<N$ ), it is known to have still a random-coil, Gaussian configuration as long as the melt chains are not too short $\left(P<N^{1 / 2}\right)$ [12]. For still shorter melt chains the $N$ chain becomes a self-avoiding walk. The radius of gyration of the longer chain is given by:

$$
\begin{aligned}
& R=a N^{1 / 2} \quad\left(P>N^{1 / 2}\right) \\
& R=a N^{3 / 5} P^{-1 / 5} \quad\left(P<N^{1 / 2}\right)
\end{aligned}
$$

Here we investigate these two cases for entangled melts $\left(P>N_{\mathrm{e}}\right)$. (Non-entangled melts $\left(P<N_{\mathrm{e}}\right)$ do not lead to such a concept as the drag number since the friction is then simply linear in $N$; this case is studied briefly in Sect. 2.2).

Let us first turn to the Gaussian tethered chain $\left(R=a N^{1 / 2}\right)$. An easy way of evaluating the drag number $X$ is to picture the $N$ chain as a random walk sequence of blobs containing 


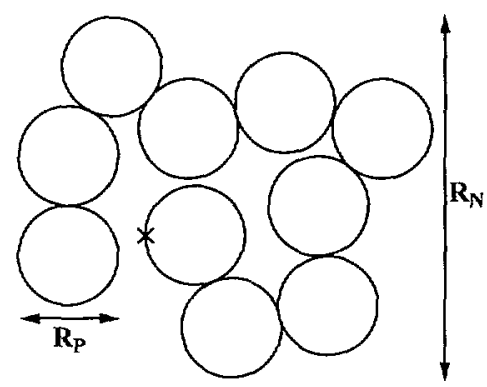

Fig. 1. - The chain $N$, immersed in a melt of shorter molecules $(P<N)$, can be seen as a random walk of blobs containing $P$ monomers. The drag number $X$ of the chain can be estimated as the sum of the blob drag numbers: $X=(N / P) X_{P}$ (Eq. (6)).

$P$ monomers (Fig. 1). Melt chains can be trapped by either section of the $N$ chain, i.e. blob contributions are additive:

$$
X=\frac{N}{P} X_{\mathrm{P}}
$$

where $X_{P}$ is the drag number of one blob of $P$ monomers. The previous results [1] yield:

$$
\begin{aligned}
& X_{P}=P / N_{e} \quad\left(P<N_{e}^{2}\right) \\
& X_{P}=P^{1 / 2} \quad\left(P>N_{\mathrm{e}}^{2}\right)
\end{aligned}
$$

Hence we simply have two regimes, depending on the melt molecular weight:

$$
\begin{aligned}
& X=N / N_{\mathrm{e}} \quad\left(P<N_{\mathrm{e}}^{2}\right) \\
& X=N / P^{1 / 2} \quad\left(P>N_{\mathrm{e}}^{2}\right)
\end{aligned}
$$

A tethered chain, swollen by an entangled melt $\left(N_{e}<P<N^{1 / 2}\right)$, is a self-avoiding walk of Gaussian blobs containing $P^{2}$ monomers; blobs here also contribute independently and we have:

$$
X=\frac{N}{P^{2}} \frac{P^{2}}{P} X_{P}
$$

Thus equations (9) and (10) hold for all $N_{\mathrm{e}}<P<N$, whether the tethered chain be Gaussian or swollen.

At higher slippage velocities, the friction force $F_{V}$ acting on a tethered chain becomes sufficient for it to elongate. It then can be seen as a sequence of blobs of size $D$ given by the Pincus law [13]:

$$
F_{V}=\frac{T}{D}
$$

The blobs are close packed in the direction of flow; perpendicular to it, the chain is still a random walk of such blobs (Fig. 2a).

More precise calculations should involve a trumpet-like conformation [14] of the chain: since friction forces due to melt chains act along the whole length of the chain, the sollicitation is higher near the attachment point and the chain should be more elongated in this region. In the present paper we do not go into such detail and we assume that $D$ is constant along the chain.

As long as each blob is larger than the melt molecules $\left(D>R_{P}\right)$, contributions are additive and again we get equations (9) and (10). 


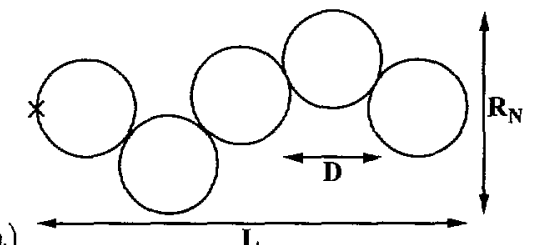

b)

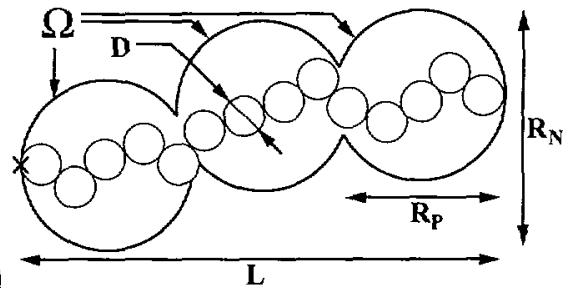

Fig. 2. - Deformed tethered chain. (a) If the blob size $D$ is larger than the melt molecular size $D>R_{P}$ ) then the blob drag numbers are additive. If, on the other hand, $D<R_{P}$ (b), one way of estimating the drag number is to compare the total number $N / N_{\mathrm{e}}$ of available entanglements and the number $n$ of melt chains present within a distance of order $R_{P}$ from the tethered chain (i.e., in the volume $\Omega=L R_{P}^{2}$ ).

When the tethered chain is even more elongated $\left(D<R_{P}\right)$, the calculation must be altered; the drag number $X$ can be estimated through the following argument: each blob is a chain of $g_{D} \equiv(D / a)^{2}$ monomers immersed in a melt of longer molecules $\left(P>g_{D}\right)$; we know [1] that the blob drag number is $X_{D}=g_{D} / N_{\mathrm{e}}$ for $g_{D}<N_{\mathrm{e}}^{2}$ and $X_{D}=g_{D}^{1 / 2}$ for $g_{D}>N_{\mathrm{e}}^{2}$. If blob contributions are additive, we get $X=\left(N / g_{D}\right) X_{D}$. Thus $X=N / N_{\mathrm{e}}$ if $g_{D}<N_{\mathrm{e}}$ and $X=N / g_{D}^{1 / 2}$ if $g_{D}>N_{e}^{2}$. This is also the result we get if we compare the total number $N / N_{\mathrm{e}}$ of available entanglements and the number $n$ of melt chains present in the vicinity of the tethered chain. Indeed $n=\Omega / P a^{3}=N / g_{D}^{1 / 2}$, where $\Omega=L R_{P}^{2}$ is the volume that contains all melt chains that might possibly be entangled with the tethered chain, i.e. all chains present within a distance $R_{P}$ from the tethered chain (the volume $\Omega$ is depicted in Fig. 2b).

As a result, we get the following expressions for the drag number of a deformed chain:

$$
\begin{aligned}
X & =N / N_{\mathrm{e}} & & \left(D>R_{P}, P<N_{\mathrm{e}}^{2}\right) \\
X & =N / P^{1 / 2} & & \left(D>R_{P}, P>N_{\mathrm{e}}^{2}\right) \\
X & =N / N_{\mathrm{e}} & & \left(D<R_{P}, g_{D}<N_{\mathrm{e}}^{2}\right) \\
X & =N / g_{\mathrm{D}}^{1 / 2} & & \left(D<R_{P}, g_{D}>N_{\mathrm{e}}^{2}\right)
\end{aligned}
$$

2.2. Grafted Surface Under Flow. - The general features of the situation of the grafted surface are recalled in the introduction: each melt chain trapped by a grafted molecule exerts a force $f_{V}(\mathrm{Eq} .(1))$ when it slides to disentangle; this gives rise to a mean force $F_{V}=X f_{V}$ (Eq. (2)) acting on each grafted chain, where $X$ is the drag number of a tethered chain, which was calculated in Section 2.1. The force $F_{V}$ determines the elongation of the grafted chain (i.e. the diameter $D$ of its blobs) via the Pincus law (Eq. (12)). The drag number $X$ may in turn depend on $D$ (Eq. (16)).

Thus, for a given melt velocity $V$ at the surface, it is possible to determine the elongation of the tethered chains and the force $F_{V}$ acting upon them, in a self-consistent manner. In the end the shear stress $\sigma$ can be calculated therefrom (Eq. (3)) and the extrapolation length $b$ can be immediately deduced from the expression of the shear rate $\left(V / b=\dot{\gamma}=\sigma / \eta_{P}\right)$.

2.2.1. Successive Regimes During Elongation. - At low velocities, the friction force $F_{V}$ is low $\left(f_{V}<T / R_{N}\right)$ and the chains remain undeformed $\left(D=R_{N}\right)$. However, when $F_{V}$ reaches $T / R_{N}$, the chains start to elongate. This corresponds to a threshold velocity $V_{1}$ given 
by $X a \eta_{P} V_{1} \equiv T / R_{N}$, i.e.:

$$
\begin{array}{rlrl}
V_{1}=\frac{T}{a^{2} \eta_{P}} \frac{N_{e}}{N^{3 / 2}} & & \left(P<N_{e}^{2}\right) \\
V_{1}=\frac{T}{a^{2} \eta_{P}} \frac{P^{1 / 2}}{N^{3 / 2}} & \left(P>N_{e}^{2}\right)
\end{array}
$$

Beyond $V_{1}$, the chains elongate smoothly. The blob diameter is given by $D=R_{N}\left(V_{1} / V\right)$, which can be written as:

$$
\begin{aligned}
D & =\frac{N_{\mathrm{e}}}{N} \frac{T}{a \eta_{P} V} & & \left(P<N_{\mathrm{e}}^{2}\right) \\
D & =\frac{P^{1 / 2}}{N} \frac{T}{a \eta_{P} V} & & \left(P>N_{\mathrm{e}}^{2}\right)
\end{aligned}
$$

For melts with a high molecular weight $\left(P>N_{\mathrm{e}}^{2}\right)$, there is a drastic change when $D$ reaches the melt radius of gyration $R_{P}$ : as can be seen from equation (16), the drag number $X$ now depends on $D$. More precisely, the force $F_{V}$ can be written in two ways:

$$
\frac{T}{D} \equiv F_{V} \equiv X a \eta_{P} V=\frac{N a^{2} \eta_{P} V}{D}
$$

This equation shows that $D$ remains undetermined and that the melt velocity is quenched at $V=V_{2}$, given by $D\left(V_{2}\right) \equiv R_{P}$, where $D$ is calculated from equation (20); hence:

$$
V_{2}=\frac{T}{a^{2} \eta_{P}} \frac{1}{N}=\frac{R_{N}}{R_{P}} V_{1} \quad\left(P>N_{\mathrm{e}}^{2}\right)
$$

Thus if $V$ is being monitored, the chains will elongate abruptly from $D=R_{P}$ to the other limit $D=a N_{\mathrm{e}}$ (i.e. $g_{D}=a N_{\mathrm{e}}^{2}$ ), when $V$ goes across the threshold slippage velocity $V_{2}$. Beyond $V_{2}$, the blob diameter $D$ is now smaller than $a N_{\mathrm{e}}$ and equation (15) applies: we recover the same regime as for the melts of low molecular weight $\left(P<N_{\mathrm{e}}^{2}\right) ; D$ is now given by equation (19).

When the blob diameter $D$ reaches Edward's tube diameter $D^{*} \equiv a N_{\mathrm{e}}^{1 / 2}$, we enter yet another regime, called the marginal regime $[3,4]$; this is for high enough velocities:

$$
V>V^{*} \equiv \frac{T}{a^{2} \eta_{P}} \frac{N_{\mathrm{e}}^{1 / 2}}{N}
$$

In this regime the grafted chain is stabilized at a fixed elongation $\left(D=D^{*}\right)$ over a wide range of velocities. Indeed, the blob diameter $D$ should be smaller than $D^{*}$; but melt chains would then disentangle completely from the tethered chain, and the force $F_{V}$ acting upon it would be significantly reduced: the chain would swell again well above $D^{*}$. The marginal regime corresponds to a linear increase of the extrapolation length $b$ with the slippage velocity $V$. The overall variations of $b$ are summarized in Figure 3.

The plateau values of $b$ are given by:

$$
\begin{aligned}
b & =b_{1} \equiv \frac{1}{\nu a N^{1 / 2}} & & \left(P>N>N_{\mathrm{e}}^{2}, V<V_{1}\right) \\
b & =b_{2} \equiv \frac{P^{1 / 2}}{\nu a N} & & \left(N>P>N_{\mathrm{e}}^{2}, V<V_{2}\right) \\
b & =b_{0} \equiv \frac{N_{\mathrm{e}}}{v a N} & & \text { (all other plateaux) }
\end{aligned}
$$


a)

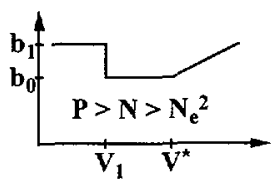

b)

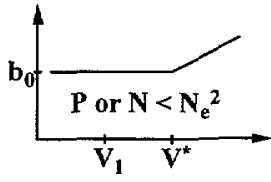

c)

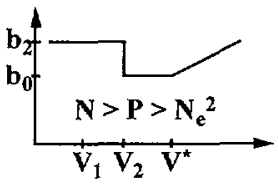

Fig. 3. - The variations of the extrapolation length versus the slippage velocity $b(V)$ are pictured for different melt $(P)$ and grafted $(N)$ chain molecular weights, relative to the threshold molecular weight $N_{\mathrm{e}}^{2}$. Situation (a) $\left(P>N>N_{\mathrm{e}}^{2}\right)$ was described earlier [4]; situations (b) and (c) are studied in the present paper. The abrupt drops of $b$ should be observable: the ratio of both plateau values is $\left(N / N_{\mathrm{e}}^{2}\right)^{1 / 2}$ for $P>N>N_{\mathrm{e}}^{2}(\mathrm{a})[4]$ and $\left(P / N_{\mathrm{e}}^{2}\right)^{1 / 2}$ for $N>P>N_{\mathrm{e}}^{2}(\mathrm{c})$.

where $\nu$ still denotes the grafting density.

We also expect that at very high slippage velocities:

$$
V>V^{* *}=\frac{T}{a^{2} \eta_{P}} \frac{1}{N_{\mathrm{e}}^{1 / 2}},
$$

the melt should behave like a rubber. This happens when the time taken by melt chains to slide out of the grafted chains becomes comparable to their reptation time, as explained elsewhere [4].

Note that the velocity $V_{1, P}$ above which trapped melt chains deform, given by

$$
f_{V}\left(V_{1, P}\right) \equiv T / R_{P}
$$

is larger than all other threshold velocities except $V^{<*}$ This effect takes place only in the marginal regime $\left(D=D^{*}\right)$ and does not change its characteristics, since the force $F_{V}$ is quenched at $F_{V}^{*} \equiv T / D^{*}$.

Among these threshold velocities, only $V_{2}$ (for high molecular weight melts, $P>N_{\mathrm{e}}^{2}$ ) and $V^{* *}$ can be obseved by macroscopic measurements, as can be seen from Figure $3 . V_{1}$ could be observed only for particular polymer systems (e.g. with chains whose monomers bear longitudinal dipoles) through techniques that should be able to show orientational order at the interface. The behavior of swollen, grafted chains $\left(N_{\mathrm{e}}<P<N^{1 / 2}\right)$ is slightly different but no directly observable quantities are changed (see Appendix A).

2.2.2. Non-Entangled Melt. - If the chain is immersed in a non-entangled melt, the force acting on the chain arises only through the straight-forward monomer-monomer friction:

$$
F_{V}=N \zeta_{1} V \approx N a \eta_{1} V
$$

For $P=N_{e}$, this expression crosses over to the entangled melt force:

$$
F_{V}=\frac{N}{N_{\mathrm{e}}} a \eta_{P} V=N \frac{P^{3}}{N_{\mathrm{e}}^{3}} a \eta_{1} V
$$

The force thus does not depend on the melt molecular weight for $P<N_{\mathrm{e}}$. If the tethered chain is swollen, there can be even fewer entanglements and the force arises only through the Rouse friction as before. Thus for the entire range $P<N_{\mathrm{e}}$, equation (29) is valid.

2.2.3. Cooperative Effect. - At high grafting densities we expect, as for $P>N[4]$, a cooperative effect to occur: melt chains should entangle with more than one tethered blob or chain. 
There should therefore be fewer entangled melt chains, and hence higher slippage velocities should be necessary to reach the same deformations of the grafted chains.

To describe this effect, we consider the surface number density $\nu_{P}$ of melt chains trapped by the grafted surface. In the low grafting density limit we have:

$$
\nu_{P}=\nu X
$$

For higher grafting densities, $\nu_{P}$ reaches a plateau. Indeed, it cannot exceed the number of melt chains present within a distance $R_{N}$ from the solid surface:

$$
\nu_{P}<\nu_{P} \max =\frac{R_{N}}{P a^{3}}=\frac{1}{a^{2}} \frac{N^{1 / 2}}{P}
$$

The crossover between equations (31) and (32) yields the threshold grafting density. For chains that are not too long (Eq. (13)) or at high velocities (Eq. (15)), the drag number $X$ is equal to $N / N_{\mathrm{e}}$ and we get:

$$
\nu_{\mathrm{c}}=\frac{1}{a^{2}} \frac{N_{\mathrm{e}}}{P N^{1 / 2}}
$$

On the other hand, for melts of high molecular weight (Eq. (14)), we get:

$$
\nu_{\mathrm{c} 2}=\frac{1}{a^{2}} \frac{1}{\sqrt{N P}}
$$

(These results can also be obtained from detailed scaling calculations of the probability that a melt chain $P$ is trapped by some grafted chain, given that it lies within a distance $R_{N}$ from the solid surface).

These threshold grafting densities $\nu_{\mathrm{c}}$ and $\nu_{\mathrm{c} 2}$ are smaller than $1 / N^{1 / 2} a^{2}$. Hence, the grafted chains may overlap $\left(\nu>1 / R_{N}^{2}\right)$ but they are not stretched away from the surface yet [15]: the calculations of the drag number $X$ remain valid.

When $\nu_{P}=\nu_{P} \max \left(\right.$ for $\nu>\nu_{\mathrm{c}}$ or $\nu_{\mathrm{c} 2}$ ), the extrapolation length $b$ reaches its lowest value: the shear stress is given by $\sigma_{\max }=\nu_{P} f_{V}=\nu_{P \max } a \eta_{P} V$. Hence:

$$
b_{\text {Inin }}=\frac{\eta_{P} V}{\sigma_{\max }}=a \frac{P}{N^{1 / 2}}=\frac{R_{P}^{2}}{R_{N}}
$$

(This value is even lower than the value $b_{\min }=R_{P}$ obtained for $N<P$ [4]).

2.2.4. Grafting Density Equivalent to Bare Solid. - In highly entangled melts $\left(P \gg N_{\mathrm{e}}\right)$ the force transmitted through the grafted chains is much higher than the mere friction of the melt chains on the solid surface and equation (3) can be harmlessly replaced by:

$$
\sigma=\nu F_{V}
$$

For shorter melt chains or non-entangled melts, however, the first term of equation (3) may dominate. The flow is then unaffected by the presence of grafted chains and the surface is equivalent to a bare solid. We explain this issue in detail below.

For non-entangled melts $\left(P<N_{\mathrm{e}}\right.$ ) the shear stress (Eqs. (3) and (29)) can be written as:

$$
\sigma=\frac{\eta_{1}}{a} V\left(1+\frac{\nu}{\nu_{\mathrm{m}}}\right)
$$

where $\nu_{\mathrm{m}}=1 / R_{N}^{2}$ is the mushroom overlap grafting density. For small grafting densities:

$$
\nu<\nu_{\text {bare }}=\nu_{\mathrm{m}}=\frac{1}{N a^{2}}
$$


the surface consequently behaves like a bare solid.

For higher molecular masses $\left(P>N_{e}\right)$ we find:

$$
\begin{array}{ll}
\nu_{\text {bare }}=\frac{N_{\mathrm{e}}^{3}}{P^{3}} \nu_{\mathrm{m}}=\frac{N_{\mathrm{e}}^{3}}{P^{3}} \frac{1}{N a^{2}} & \left(X=N / N_{\mathrm{e}}\right) \\
\nu_{\text {bare }}=\frac{N_{\mathrm{e}}^{2} N^{1 / 2}}{P^{3}} \frac{1}{N a^{2}} \quad\left(X=N / P^{1 / 2}, g_{D}>P>N_{\mathrm{e}}^{2}\right)
\end{array}
$$

Thus, if $P$ is well above $N_{\mathrm{e}}$, the grafted chains do affect the flow (except if we manage to achieve extremely scarce grafting).

\section{The Total Entanglement Molecular Weight Threshold}

As stated in the introduction, the binary entanglement model leads to two different regimes for the number $X$ of chains trapped by a given chain [1]. This number is the total number of chains that have to be removed from the vicinity of the chain under consideration in order to renew all topological constraints that act upon it.

The number of chains trapped by a short chain should be linear in $N$ :

$$
X=\frac{N}{N_{\mathrm{e}}} \quad\left(N<N_{\mathrm{e}}^{2}\right)
$$

Longer chains should trap virtually all chains present in their vicinity:

$$
X=N^{1 / 2} \quad\left(N>N_{\mathrm{e}}^{2}\right)
$$

According to predictions based on this model (for the case $P>N[4]$ and for the opposite case in the present paper), long chains $\left(N>N_{\mathrm{e}}^{2}\right)$ should display both regimes, depending on the slippage velocity, and large variations of some quantities such as the extrapolation length $b$ consequently might be seen in shear flow slippage experiments such as those already mentioned [10]. We wish to discuss here whether the threshold $N=N^{\times} \equiv N_{\mathrm{e}}^{2}$ can be reached for real polymers.

3.1. The Threshold Molecular Weight. - Consider a particular flexible polymer of molecular weight $M$ in the liquid state and let $M_{\mathrm{e}}^{\prime}$ be the entanglement mass (the molecular mass above which it displays entanglement effects). The determination of $M_{\mathrm{e}}^{\prime}$ can be achieved by several methods (with some discrepancies) such as viscosity, elastic plateau modulus, steady-state compliance or self-diffusion constant measurements [6,16]. A straight-forward transcription for the threshold molecular mass $M^{*}$ corresponding to $N^{*}=N_{\mathrm{e}}^{2}$ would lead to:

$$
\left(M^{\times} / m\right) \equiv\left(M_{\mathrm{e}}^{\prime} / m\right)^{2}
$$

where $m$ denotes the average molecular weight of a main chain link. This result is indeed what we find below for an ideally flexible polymer (whose persistence length $l_{\mathrm{p}}$ is equal to the average length $l_{0}$ of a main chain link, or equivalently whose characteristic ratio $C_{\infty}$ [17] is equal to unity, and whose monomers are roughly spherical). For real polymers, however, a closer look at this threshold mass is necessary. Calculations, reported below, yield the following expression for $M^{*}$ :

$$
\left(\Lambda M^{*} / m\right) \equiv\left(M_{\mathrm{e}}^{\prime} / m\right)^{2}\left(\frac{\rho}{m / N_{\mathrm{A}}}\left(\sqrt{l_{0} l_{\mathrm{p}}}\right)^{3}\right)^{2}
$$


where $\rho$ denotes the melt viscosity. $N_{\mathrm{A}}$ is Avogadro's number. The last factor is the square of the number of monomers present in a volume of dimension $\sqrt{l_{0} l_{p}}$; this number is unity if $l_{\mathrm{p}} \equiv C^{\infty} l_{0}=l_{0}$ and if each monomer occupies a volume $l_{0}^{3}$.

Some quantities may seem redundant in equation (44). In particular, one might think that $M_{\mathrm{e}}^{\prime}$ could be expressed in terms of $m, \rho, l_{0}$ and $C_{\infty} \equiv l_{\mathrm{p}} / l_{0}$. However this question alone represents an entire field of research. Semi-empirical laws have been established [18] but no thorough theory has yet been constructed, although various approaches have been proposed [19-21]. Here we do not address this question at all. We instead use the values of these parameters (including $M_{\mathrm{e}}^{\prime}$ ) available in the literature; indeed, they can be measured independently for a given polymer.

To evaluate the threshold mass $M^{\star}$, we can use two approaches [1]: either we globally compare the number of chains present in the vicinity of the test chain and the available number of entanglements along it, or we look more precisely at inter-chain binary contacts, some of which constitute entanglements.

3.1.1. First Approach: Global Comparison. - The number of entanglements acting on the test chain is $M / M_{\mathrm{e}}^{\prime}$. Due to the binary entanglement hypothesis, this is also supposed to be the number of times that a binary contact with some other chain is actually an entanglement. The radius of gyration of the test chain is given by:

$$
R=l_{\mathrm{p}}\left(\frac{M}{C_{\infty} m}\right)^{1 / 2}=l_{0}\left(\frac{C_{\infty} M}{m}\right)^{1 / 2}
$$

The number of chains present in the vicinity of the test chain is of order:

$$
n\left(R^{3}\right)=\frac{\rho R^{3}}{M / N_{\mathrm{A}}}=\frac{\rho l_{0}^{3} N_{\mathrm{A}} C_{\infty}^{3 / 2} M^{1 / 2}}{m^{3 / 2}}
$$

If $M / M_{\mathrm{e}}^{\prime}<n\left(R^{3}\right)$, we expect that about $M / M_{\mathrm{e}}^{\prime}$ chains will be entangled with the test chain each of which having in general only one entanglement with it. If the reverse is true, then almost all of the $n\left(R^{3}\right)$ chains present will be trapped: each will make on average $\left(M / M_{\mathrm{e}}^{\prime}\right) / n\left(R^{3}\right)$ entanglements with the test chain.

The threshold molecular mass is given by $M^{\star} / M_{\mathrm{e}}^{\prime} \equiv n\left(R^{3}\left(M^{\times}\right)\right)$; this condition leads to:

$$
M^{\star}=\frac{\rho^{2} l_{0}^{6} M_{e}^{\prime 2} N_{\mathrm{A}}^{2} C_{\infty}^{3}}{m^{3}}
$$

which is equivalent to equation (44).

3.1.2. Second Approach: Binary Contacts. - The mean spatial distance between consecutive entanglements along the test chain is:

$$
R_{\mathrm{e}}=l_{0}\left(\frac{C_{\infty} M_{e}^{\prime}}{m}\right)^{1 / 2}
$$

Approximately $n\left(R_{\mathrm{e}}^{3}\right)$ other chains pass through this volume, but only one is entangled with the test chain. Hence the probability that two chains are entangled when they are as close to one another as $R_{\mathrm{e}}$ is:

$$
p=\frac{1}{n\left(R_{\mathrm{e}}^{3}\right)}
$$


Any chain passing in the vicinity of the test chain comes about $\left(M / M_{\mathrm{e}}^{\prime}\right)^{1 / 2}$ times close to it (i.e., at a smaller distance than $R_{e}$ ). Thus it is entangled about $c$ times with the test chain, where $c$ is given by:

$$
c=\left(M / M_{\mathrm{e}}^{\prime}\right)^{1 / 2} p=\frac{M^{1 / 2} m^{3 / 2}}{M_{\mathrm{e}}^{\prime} \rho l_{0}^{3} N_{\mathrm{A}} C_{\infty}^{3 / 2}}
$$

If $c$ is greater than unity, then virtually all $n\left(R^{3}\right)$ chains are entangled (on average) $c$ times with the test chain. If $c$ is smaller than unity, each of the $n\left(R^{3}\right)$ chains has the probability $c$ to be entangled with the test chain. The number of trapped chains in this case $(c<1)$ is therefore:

$$
X=c n\left(R^{3}\right)=\frac{M}{M_{\mathrm{e}}^{\prime}}
$$

Thus, the threshold mass $M^{*}$ is given by $c\left(M^{*}\right) \equiv 1$, and this again yields equation (44).

3.1.3. Numerical Values and Discussion. -- Values for $M^{*}$ were compiled for various polymers using equation (44) and are presented in Table I. They were calculated either with $M_{\mathrm{e}}^{\prime} \equiv M_{\mathrm{e}}$ given by elastic plateau modulus measurements or with $M_{\mathrm{e}}^{\prime} \equiv M_{\mathrm{c}}$, where $M_{\mathrm{c}}$ denotes the critical mass between linear and entangled viscosity regimes. Another quantity $\alpha$ was introduced, given by:

$$
\alpha \equiv(\nu L)^{-1 / 2} / l_{\mathrm{p}}
$$

where $\nu L$ is the contour length concentration [18]. As explained below in Section $3.2, \alpha$ is a measure of the local, rod-like or random coil-like, aspect of the chains. Quantities in brackets were not present in the data used and were put in for the computation of $M^{\times}$.

Three remarks must be made.

- Values of $M^{\star}$ calculated from $M_{\mathrm{e}}$ and from $M_{\mathrm{c}}$ sometimes differ by large factors (up to more than 60). It is not clear which one is the more relevant for our concern.

- In the binary constraint model recalled above, we consider that one entanglement is caused by the presence of one other chain. It may appear more realistic to demand that several chains be present; this would lead to smaller values of $M^{*}$.

- Some systems (star polymers) should exhibit strong entanglement (Stokes) regime, although with objects whose sizes correspond to molecular weights lower than $M^{*}$.

The last two points are now discussed.

3.2. Multiple Binary Constraint Entanglement Model. -- The binary entanglement model [1] recalled above supposes that entanglements arise only through binary topological constraints. In other words, situations like that depicted in Figure 4a (chain 2 alone imposes a topological constraint upon chain 1) are supposed to be much more frequent than that of Figure $4 \mathrm{~b}$ (both chains 2 and 3 have to be present for chain 1 to feel a constraint).

Furthermore, it was assumed in the model that one such constraint could build up an entanglement (more precisely, that renewing one given entanglement along a chain required that one particular other chain be removed). However, we may think that a few such constraints are necessary to build up an entanglement. Lin [19] suggested that a fixed number of chains (about 18 ) should be present in a volume $R_{\mathrm{e}}^{3}$ (given by Eq. (48)) and that this condition defines $M_{\mathrm{e}}^{\prime}$; it appears to be based on the assumption that about four chains must be present to impose an entanglement on a given chain. Here we propose a detailed, though not thoroughly quantitative approach of the required number of binary constraints and we look at the consequent reduction of the predicted molecular mass threshold $M^{\star}$. 
Table I. - Characteristics of some current polymers are shown. Where available, the entanglement mass issued by both elastic modulus $\left(M_{\mathrm{e}}\right)$ and viscosity measurements $\left(M_{\mathrm{c}}\right)$ were indicated. The corresponding threshold molecular mass $M^{*}$ is calculated from equation (47).

\begin{tabular}{|c|c|c|c|c|c|c|c|c|c|c|c|}
\hline Species & $\begin{array}{l}m \\
\mathrm{~g} / \mathrm{mol}\end{array}$ & ref & $\begin{array}{l}l_{0} \\
\AA\end{array}$ & $C_{\infty}$ & $\begin{array}{l}T \\
K\end{array}$ & $\begin{array}{l}M_{\mathrm{e}} \\
8 / \mathrm{mol}\end{array}$ & $\begin{array}{l}M_{\mathrm{c}} \\
\mathrm{g} / \mathrm{mol}\end{array}$ & $\begin{array}{l}\rho \\
\mathrm{g} / \mathrm{cm}^{3}\end{array}$ & $\begin{array}{l}M_{\mathrm{e}}^{*} \\
\mathrm{~kg} / \mathrm{mol}\end{array}$ & $\begin{array}{l}M_{\mathrm{c}}^{*} \\
\mathrm{~kg} / \mathrm{mol}\end{array}$ & $\alpha$ \\
\hline \multirow[t]{3}{*}{$\overline{\mathrm{PE}}$} & 14 & 22 & 154 & 7.0 & & & 5096 & $(0.802)$ & & 14800 & \\
\hline & & 18 & 1.54 & 7.0 & 463 & & 3800 & 0.76 & & 5000 & 4.1 \\
\hline & & 19 & 1.54 & 7.2 & 373 & 737 & & 0.802 & 230 & & 3.9 \\
\hline \multirow[t]{3}{*}{ PYAC } & 17 & 22 & 1.54 & 9.4 & & & 24500 & $\left(\begin{array}{ll}1 & 14\end{array}\right)$ & & 39000 & 44 \\
\hline & & 18 & 154 & 9.0 & 330 & & 24500 & 114 & & 35000 & 46 \\
\hline & & 19 & 154 & 9 & 330 & 6950 & & 114 & 2800 & & 4.6 \\
\hline \multirow[t]{2}{*}{ HPIP } & 175 & 22 & 154 & 6.8 & & & 3970 & $(0.854)$ & & 3300 & 45 \\
\hline & & 19 & 154 & 6.8 & 298 & 1472 & & 0.854 & 450 & & 45 \\
\hline \multirow[t]{3}{*}{ P $\alpha$ MS } & 59 & 22 & 154 & 10.5 & & & 28000 & $\left(\begin{array}{ll}1 & 04\end{array}\right)$ & & 49000 & 4.8 \\
\hline & & 18 & 154 & 10.1 & 473 & & 40700 & 1.04 & & 21000 & 50 \\
\hline & & 19 & 154 & 10.1 & 473 & 10225 & & 1.04 & 2700 & & 50 \\
\hline \multirow[t]{3}{*}{ PS } & 52 & 22 & 154 & 10.0 & & & 35000 & $(1.0)$ & & 42000 & 49 \\
\hline & & 18 & 154 & 10.3 & 463 & & 35000 & 0.97 & & 43000 & 4.8 \\
\hline & & 19 & 1.54 & 9.4 & 400 & 13500 & & 1.007 & 5300 & & 5.2 \\
\hline HPBD $(043)$ & 178 & 19 & 1.54 & 6.4 & 338 & 1700 & & 0.832 & 450 & & 49 \\
\hline \multirow[t]{3}{*}{ PMMA } & 50 & 22 & 1.54 & 7.0 & & & 31500 & $\left(\begin{array}{ll}1 & 14\end{array}\right)$ & & 17100 & 6.4 \\
\hline & & 18 & 154 & 87 & $\begin{array}{l}473- \\
423\end{array}$ & & 31000 & 1.14 & & 32000 & 51 \\
\hline & & 19 & 154 & 87 & $\begin{array}{l}473- \\
423\end{array}$ & 6176 & & 114 & 1300 & & 5.1 \\
\hline $\operatorname{PBD}(043)$ & 17.2 & 19 & 147 & 6.1 & 289 & 1860 & & 0.9 & 460 & & 5.2 \\
\hline PBD (0 99) & 26.7 & 19 & 154 & 7 & 306 & 4280 & & 0.883 & 1200 & & 53 \\
\hline \multirow[t]{2}{*}{ PPO } & $(145)$ & 22 & 1.49 & 51 & & & 5800 & $\left(\begin{array}{lll}1 & 00\end{array}\right)$ & & 5800 & 5.3 \\
\hline & & 18 & 1.49 & 5.1 & 298 & & 5800 & 100 & & 5800 & 5.3 \\
\hline \multirow[t]{2}{*}{ PBD (c1s) } & 135 & 22 & 1.47 & 5.15 & & & 5900 & $(09)$ & & 5800 & 5.4 \\
\hline & & 19 & 1.47 & 4.9 & 298 & 2347 & & 09 & 780 & & 5.7 \\
\hline $\operatorname{PBD}(0.08)$ & 14.1 & 19 & 1.47 & 5.1 & 300 & 1430 & & 0896 & 280 & & 5.6 \\
\hline \multirow[t]{2}{*}{$\mathrm{PP}$} & (21) & 22 & 1.54 & 6.2 & & & 6900 & & & 3300 & 58 \\
\hline & & 18 & 1.54 & 5.8 & 463 & & 7000 & 075 & & 2800 & 6.2 \\
\hline \multirow[t]{3}{*}{ PIP (cis) } & 17 & 22 & 147 & 5.0 & & & 7650 & $(0.9)$ & & 4400 & 63 \\
\hline & & 18 & 147 & 5.3 & $\begin{array}{l}298- \\
243\end{array}$ & & 10000 & 090 & & 9000 & 5.9 \\
\hline & & 19 & 147 & 5.3 & 298 & 4054 & & 09 & 1480 & & 59 \\
\hline \multirow[t]{3}{*}{ PIB } & 28 & 22 & 1.54 & 50 & & & 16000 & $(089)$ & & 5600 & 76 \\
\hline & & 18 & 1.54 & 6.2 & 298 & & 15200 & 0.89 & & 9600 & 61 \\
\hline & & 19 & 154 & 6.2 & 298 & 7056 & & 0.89 & 2100 & & 6.1 \\
\hline \multirow[t]{2}{*}{ PHMA } & 85 & 22 & 1.54 & 10.0 & & & 91800 & $(0.95)$ & & 60000 & 64 \\
\hline & & 19 & 154 & 10.3 & 373 & 27090 & & 095 & 5700 & & 62 \\
\hline \multirow[t]{3}{*}{$\mathrm{PEO}$} & 14.6 & 22 & 1.49 & 4.2 & & & 4380 & $\left(\begin{array}{ll}1 & 08\end{array}\right)$ & & 2100 & 62 \\
\hline & & 18 & 1.49 & 3.8 & 298 & & 3600 & 108 & & 1060 & 69 \\
\hline & & 19 & 1.49 & 38 & 298 & 1500 & & $(1.08)$ & (184) & & 6.9 \\
\hline \multirow[t]{2}{*}{ PEBMA } & 85 & 22 & & 9.3 & & & 42800 & $(0.995)$ & & 11500 & 67 \\
\hline & & 19 & 1.54 & 9.1 & 373 & 17632 & & 0.995 & 1800 & & 6.8 \\
\hline \multirow[t]{2}{*}{ POMA } & 99 & 22 & 1.54 & 10.0 & & & 114000 & $(0927)$ & & 56000 & 70 \\
\hline & & 19 & 1.54 & 10 & 373 & 69691 & & 0927 & 21000 & & 70 \\
\hline HPBD (0 99) & 27.7 & 19 & 1.54 & 55 & 373 & 10694 & & 0.819 & 2900 & & 7.1 \\
\hline \multirow[t]{3}{*}{ PDMS } & 37 & 22 & 146 & 6.0 & & & 24420 & & & 8400 & 7.5 \\
\hline & & 18 & 146 & 5.2 & & & 24500 & 097 & & 5500 & 87 \\
\hline & & 19 & 1.46 & 5.2 & 298 & 8010 & & 0.97 & 590 & & 8.7 \\
\hline
\end{tabular}

In polymer solutions and melts, the contour length concentration $\nu L$ as defined by Graessley and Edwards [18] is a crucial parameter $\left(\nu L=l_{0} \rho N_{\mathrm{A}} / m\right.$ in the present notations). $(\nu L)^{-1 / 2}$ is the characteristic length scale of inter-chain distances. Thus, if $(\nu L)^{-1 / 2} \ll l_{\mathrm{p}}$ (i.e. $a \ll 1$ ), chains interact locally as rigid rods, whereas if $(\nu L)^{-1 / 2} \gg l_{\mathrm{p}}$ (i.e. $\alpha \gg 1$ ), they interact as random coils. Let us first consider the local rigid rod limit (high contour length concentration). Due to the presence of three other chains, the white chain depicted in Figure 5a has no more than one translational degree of freedom (reptation) and still has (part of) both rotational degrees of freedom (we forget about the rotation about its own axis). If we put two or three other chains (Fig. 5b), we can remove all degrees of freedom, but reptation. Thus the presence 


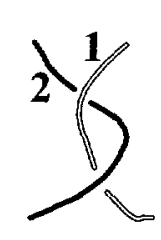

a)

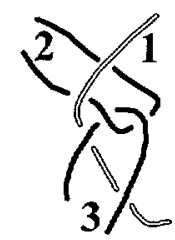

b)

Fig. 4. - The white chain undergoes a topological constraint (it cannot move to the right). In situation (a), chain 2 alone exerts the constraint. In situation (b), both chains 2 and 3 have to be present: if any one of them slides away, the constraint upon chain 1 is released. The binary entanglement hypothesis stipulates that situations like (b) are very rare as compared to (a).

a)

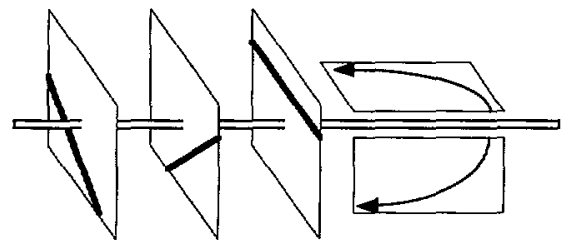

b)

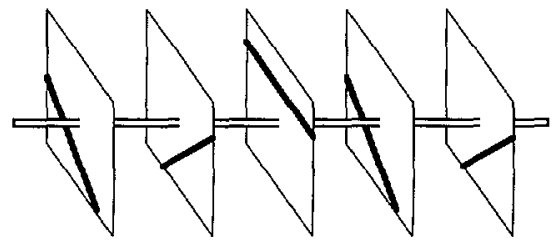

Fig. 5. - The white rod still has part of two rotational degrees of freedom if three other rods (a) are present (we ignore rotation about its own axis). If two more rods are properly arranged (b), it now only can be translated along its own axis. This leads to the value $z_{\text {rod }} \approx 5$ (Eq. (53)).

of $z_{\text {rod }}$ other chains can give rise to one entanglement, with:

$$
z_{\text {rod }} \approx 5
$$

At lower contour length concentrations, chains interact as Gaussian random coils. Let $z_{\text {coil }}$ be the number of constraints required to produce one entanglement (i.e., the number that corresponds to $z_{\text {rod }}$, but in the Gaussian case). The question of whether $z_{\text {coil }}$ is smaller or greater than $z_{\text {rod }}$ is not clear. On the one hand, Gaussian chains are rougher than rigid rods and each chain may exert constraints on the test chain in several directions at a time; $z_{\text {coil }}$ may thus be smaller than $z_{\text {rod }}$. On the other hand, the conformation of Gaussian chains cannot be considered as frozen except on very short time scales; thus more chains may have to be present, and this would make $z_{\text {coil }}$ larger. $z_{\text {coil }}$ might be tractable by numerical means. Anyhow. it would probably lie between $0.5 z_{\text {rod }}$ and $2 z_{\text {rod }}$.

Now if we turn back to the mass threshold problem, $M / M_{e}^{\prime}$ entanglements now correspond to $z M / M_{\mathrm{e}}^{\prime}$ chains present and trapped by the test chain, where $z$ is the relevant factor $\left(z=z_{\text {rod }}\right.$ or $\left.z_{\text {coll }}\right)$. Retracing back the calculations presented above, we have to replace equation (44) with:

$$
M^{\times} \equiv m \frac{1}{z^{2}}\left(M_{\mathrm{e}}^{\prime} / m\right)^{2}\left(\frac{\rho}{m / N_{\mathrm{A}}}\left(\sqrt{l_{0} l_{\mathrm{p}}}\right)^{3}\right)^{2}
$$

The value of $z^{2}$, according to the above considerations, should lie between 5 and possibly 100 . The numerical values of $M^{*}$ calculated above then should be divided by this factor. The mass threshold might then be within much easier reach.

3.3. Surface Grafted with Star-Shaped Polymers. - As described above, the mass threshold between linear entanglement (Rouse regime, Eq. (41)) and total trapping (Stokes 
a)

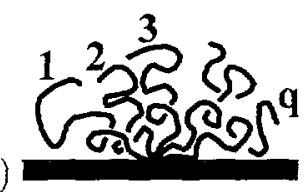

b)

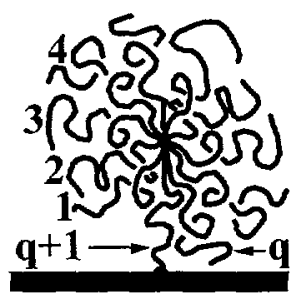

c)

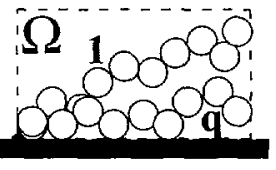

Fig. 6. -- Grafted star-shaped polymers immersed in a melt of linear molecules should lead to lower mass thresholds (for total entanglement) than for linear grafted chains. Star molecules could be attached either by their centers (a) or by their arm-ends (b). Deformed stars occupy a volume $\Omega \equiv L R_{N}^{2}$ (c).

regime, Eq. (42)) can be understood as the molecular mass for which the number of chains present with in the radius of gyration of the test chain is of the same order as the available number of entanglements along it.

In order to increase the number of entanglement points without significantly changing the size of the molecule, a simple idea is to use star-shaped molecules. One may think of at least two ways of grafting such star molecules ( $q$ linear arms, $N$ monomers each): we may attach the branching point to the solid surface (Fig. 6a) or we may use one arm to fix the star to the surface (Fig. 6b). The second situation might be easier to achieve in practice with a reasonable distribution of $q$; for small $q$, however, it should display a number of intermediate regimes, due to the elongation of the grafted arm. For the sake of simplicity, we here look at the easier situation: all $q$ arms are supposed to be attached to the same point on the solid surface (Fig. 6a).

The radius of gyration $R$ of a star molecule has different expressions for various arm lengths $N$, melt molecular weights $P$ and arm numbers $q[23]$ :

$$
\begin{aligned}
& R=a N^{1 / 3} q^{1 / 3} \quad\left(q>N^{1 / 2}, q^{2} P^{3}>N^{4}\right) \\
& R=a N^{1 / 2} \quad\left(q<N^{1 / 2}, N q^{2}<P^{2}\right) \\
& R=a N^{3 / 5} q^{1 / 5} P^{-1 / 5} \quad\left(q^{2} P^{3}<N^{4}, N q^{2}>P^{2}\right)
\end{aligned}
$$

The drag number $X$ of a star at rest (i.e., at small melt velocities, when the force acting on the star does not elongate it) can be estimated by scaling arguments similar to those described in Section 2.1. For melts of high molecular weight $\left(R_{P}>R\right.$, where $R_{P}=a P^{1 / 2}$ denotes the radius of gyration of the melt molecules), we find:

$$
X=\min \left\{\frac{R}{a}\left(1-\frac{q N a^{3}}{R^{3}}\right), \frac{q N}{N_{\mathrm{e}}}\right\} \quad\left(R_{P}>R\right)
$$

where $R$ is given by equation $(56)$ or $(57)$. or melts of lower molecular weight, $\left(R_{P}<R\right)$ we get:

$$
X=\min \left\{\frac{R^{3}}{P a^{3}}\left(1-\frac{q N a^{3}}{R^{3}}\right), \frac{q N}{P^{1 / 2}}, \frac{q N}{N_{e}}\right\} \quad\left(R_{P}<R\right)
$$

Of course, dense stars (Eq. (55)) have a drag number that is essentially zero, since melt chains do not penetrate the star and cannot entangle with it.

The regimes for the drag number are summarized in Figure 7 . The particular case $q=1$ again yields the previous results (see Sect. 2.1 and the opposite case [4] $P>N$ ). For melt 
a)

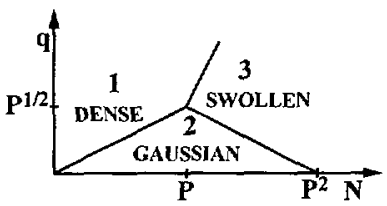

b)

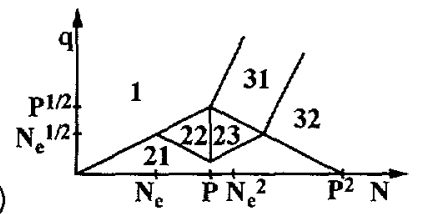

c)

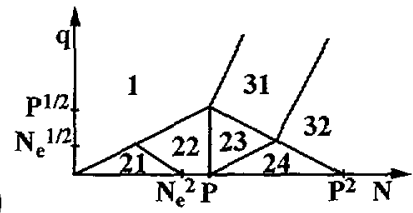

Fig. 7. - Star-shaped polymer with $q$ arms of $N$ monomers each, immersed in a melt with molecular weight $P$, are globally dense (region 1), Gaussian (2) or swollen (3). But they always have a dense core, and swollen stars with $q<P^{1 / 2}$ also have an intermediate, Gaussian layer [23]. The expressions for the drag number of a star are the following: $X \cong 0$ (region 1$), X \cong q N / N_{e}(21,32 \mathrm{~b}), X \cong N^{1 / 2}$ (22), $X \cong N^{3 / 2} / P(23), X \cong N^{9 / 5} q^{3 / 5} P^{-8 / 5}(31), X \cong q N / P^{1 / 2}(24,32 \mathrm{c})$.

molecular weights lower than the total entanglement threshold $\left(P<N_{\mathrm{e}}^{2}\right.$ ), stars can trap all chains present, provided they have a sufficient number of arms (regions 22, 23 and 31 ). The behavior of a surface grafted with stars in these regimes should be similar to that of a surface grafted with long, linear molecules in a melt of high molecular weight $\left(P, N>N_{\mathrm{e}}^{2}\right)$. This is calculated in Appendix $B$ for stars with a moderate number of arms (regions 22 and 23).

These regimes do not require that the melt molecules or the star arms be longer than the threshold molecular weight. For instance, if $P=N$ and $N_{\mathrm{e}} / P^{1 / 2}<q<N_{\mathrm{e}}^{1 / 2}$, then $b$ changes by a factor $q N^{1 / 2} / N_{\mathrm{e}}$ when $V \approx V_{1}$, and this factor can be as high as 10 if $N$ is about 100 times above the entanglement number $N_{\mathrm{e}}$ : this feature of $b(V)$ should be observable.

\section{Conclusion}

The study of the shear flow of a polymer melt on a solid surface grafted with polymer molecules that are shorter [4] or longer than the melt chains (in the present paper), led to a new point of view about the drag number $X$, which is the number of melt chains entangled with a tethered chain [1]: this number should have a different dependence on molecular weight, according to whether the chains used are longer or shorter than some threshold molecular weight $M^{*}$.

The existence of this threshold and of both regimes is still a hypothesis. In the present paper we tried to evaluate precisely the value of the threshold molecular weight as a function of the known parameters of the polymer under consideration; of course, the model suffers from an undetermined global numerical coefficient, due to the scaling approach used and to the various reasons discussed in Sections 3.1.3 and 3.2. However, the numerical factors should be roughly the same for all (linear) polymers, so that - if the threshold does have some reality - the relative ratios of the numerical values given in Table I should remain true.

We also indicated a system involving grafted star-shaped polymers, which should enable us to lower the molecular weight threshold by a known factor; this would allow for a test of this model, since the predicted behaviors below and above the threshold are qualitatively different.

\section{Acknowledgments}

I am much indebted to Pierre-Gilles de Gennes and to Elie Raphaël for stimulating discussions, and to both them and John Folkers for a critical reading of the manuscript. 


\section{Appendix A}

\section{Swollen, Grafted Chains}

A long, tethered chain $\left(N>N_{\mathrm{e}}^{2}\right)$ can be swollen by an entangled melt $\left(N_{e}<P<N^{1 / 2}\right)$. It is then, in fact, Gaussian at small length scales $\left(g^{1 / 2}<P\right)$. It has thus the structure of a self-avoiding walk of Gaussian blobs of $P^{2}$ monomers.

We verify:

$$
R=\left(a\left(P^{2}\right)^{1 / 2}\right)\left(\frac{N}{P^{2}}\right)^{3 / 5}=a N^{3 / 5} P^{-1 / 5}
$$

The first factor is the size of a Gaussian blob of $P^{2}$ monomers and $N / P^{2}$ is the number of such blobs.

The drag number $X$ of a swollen grafted chain can still be expressed by equations (9), 10), (13) and (14). Indeed, their Gaussian sub-blobs of $P^{2}$ monomers are larger than $R_{P}$. However, since their radius of gyration at rest is enhanced (Eq. (5)), the velocity at which they start to elongate is reduced and equations (17) and (18) are now replaced by:

$$
\begin{aligned}
& V_{1}=\frac{T}{a^{2} \eta_{P}} \frac{N_{\mathrm{e}} P^{1 / 5}}{N^{8 / 5}} \quad\left(P<N_{\mathrm{e}}^{2}\right) \\
& V_{1}=\frac{T}{a^{2} \eta_{P}} \frac{P^{7 / 10}}{N^{8 / 5}} \quad\left(P>N_{\mathrm{e}}^{2}\right)
\end{aligned}
$$

At slippage velocities higher than $V_{1}$, the chains elongate according to $D=R\left(V_{1} / V\right)$, where $R$ is given by equation (5). When they are further elongated, the diameter $D$ of their blobs reaches the size $a\left(P^{2}\right)^{1 / 2}$ of the Gaussian sub-blobs.

This happens for $V=V_{1}^{\prime}$, with:

$$
\begin{aligned}
V_{1}^{\prime} & =\frac{T}{a^{2} \eta_{P}} \frac{N_{\mathrm{e}}}{N P} & & \left(P<N_{\mathrm{e}}^{2}\right) \\
V_{1}^{\prime} & =\frac{T}{a^{2} \eta_{P}} \frac{1}{N P^{1 / 2}} & & \left(P>N_{\mathrm{e}}^{2}\right)
\end{aligned}
$$

Above $V_{1}^{\prime}$, the grafted chains are fully Gaussian, elongated chains: the swelling effect has been overcome by the stretching. At still higher slippage velocities, we reach the sudden stretching transition at $V_{2}$ for melts of high molecular weight $\left(P>N_{\mathrm{e}}^{2}\right)$, and the marginal regime appears above $V^{\times}$.

The threshold velocities $V_{1}$ and $V_{1}^{\prime}$ do not lead to any macroscopically abservable changes like $V_{2}$ and $V^{\times}$, as explained in the main text.

\section{Appendix B}

\section{Grafted Stars Under Flow}

Grafted linear molecules lead to strong variations of $b(V)$, provided that they and the melt chains are long enough $\left(P, N>N_{\mathrm{e}}^{2}\right)$. But the threshold molecular weight $N_{\mathrm{e}}^{2}$ might be too high for an easy check of this effect (see discussion in Sects. 3.1 and 3.2). On the other hand, the grafting of star molecules allows to avoid using so long molecules. Indeed, the threshold molecular weight can be substantially lowered for well chosen values of the parameters, as was indicated in Section 3.3. 
We here outline the calculations that lead to these results: we first indicate how to estimate the drag number of a deformed star; we then give the main features of the resulting flow characteristics.

B.1. Drag Number of a STAR. - A deformed star in the Gaussian regime (region 2 of Fig. 7a) remains within the volume $\Omega \equiv L R_{N}^{2}$ depicted in Figure $6 \mathrm{c}$. The undeformed part of the star (near the attachment point) has a drag number that is much smaller than the drag number of the rest of the star, as soon as the star is really deformed $\left(D \ll R_{N}\right)$. We here concentrate on the blobs that are away from the grafting point.

If $P<N$, we can proceed as in Section 2.1. If $D>R_{P}$, we have:

$$
X<\min \left\{\frac{q N}{N_{\mathrm{e}}}, \frac{q N}{P} P^{1 / 2}\right\}
$$

If, on the other hand, $D>R_{P}$, we have:

$$
X<\min \left\{\frac{q N}{N_{\mathrm{e}}}, \frac{q N}{g_{D}} g_{D}^{1 / 2}\right\}
$$

But if there are many arms, then another limitation will be important: all chains trapped have to be within the volume $\Omega$. Thus, we also have:

$$
X<\frac{\Omega}{P a^{3}}
$$

In the case of interest $\left(P, N<N_{\mathrm{e}}^{2}\right)$, we have the simpler expression:

$$
X=\min \left\{\frac{q N}{N_{\mathrm{e}}}, \frac{\Omega}{P a^{3}}\right\} \quad(P<N)
$$

If $P>N, P$ chains that cross the volume $\Omega$ use up about $N$ monomers in this region; condition (B.3) thus has to be replaced by $X<\Omega / N a^{3}$. We get the following drag number:

$$
X=\min \left\{\frac{q N}{N_{\mathrm{e}}}, \frac{\Omega}{N a^{3}}\right\} \quad(P>N)
$$

B.2. Flow Characteristics. - The behavior of a surface grafted with stars can be calculated from the expression of the drag number, using equations (1), (2) and (3) as before. Only the Pincus law (Eq. (12)) has to be altered, since the global force $F_{V}$ acts simultanously on all arms:

$$
F_{V}=\frac{q T}{D}
$$

Again the extrapolation length $b$ is deduced from the expression of the shear rate $(V / b=\dot{\gamma}=$ $\sigma\left(\eta_{P}\right)$. It turns out that regions 22 and 23 of Figure $7 \mathrm{~b}$ are to be divided into two parts: for stars with a moderate number of arms, $b(V)$ behaves like the curve of Figure 3 a (and the value of $V^{*}$ is unchanged and is given by equation (23)), whereas for larger $q$, the stars enter directly the marginal regime after elongating abruptly at $V \approx V_{1}$. In other words, $V^{*}=V_{1}$ for these regimes, and $b$ immediately starts to increase after it has reached its minimum value $b_{0}^{*}$ just beyond $V_{1}$.

We here give the flow characteristics. 
Region 22 $(P>N)$ :

$$
\begin{array}{rlrl}
V_{1}(q) & =\frac{T}{a^{2} \eta_{P}} \frac{q}{N} & \\
b=b_{1}=\frac{1}{\nu a N^{1 / 2}} & & \left(V<V_{1}\right) \\
b_{0}(q) & =\frac{N_{\mathrm{e}}}{\nu a N} & & \left(V_{1}<V<V^{*}, q<N_{\mathrm{e}}^{1 / 2}\right) \\
b_{\min } & =b_{0}=\frac{N_{\mathrm{e}}^{1 / 2}}{\nu a N} & & V \approx V^{\varkappa}=V_{1} \quad\left(q>N_{\mathrm{e}}^{1 / 2}\right)
\end{array}
$$

Region $23(P<N)$ :

$$
\begin{aligned}
V_{1}(q) & =\frac{T}{a^{2} \eta_{P}} \frac{q P}{N^{2}} & & \\
b=b_{1} & =\frac{P}{\nu a N^{3 / 2}} & & \left(V<V_{1}\right) \\
b_{0}(q) & =\frac{N_{\mathrm{e}}}{\nu a q N} & & \left(V_{1}<V<V^{-}, q<\frac{N_{\mathrm{e}}^{1 / 2} N}{P}\right) \\
b_{\min } & =b_{0}^{\times}=\frac{P N_{\mathrm{e}}^{1 / 2}}{\nu a N^{2}} & & V \approx V^{*}=V_{1} \quad\left(q>\frac{N_{\mathrm{e}}^{1 / 2} N}{P}\right)
\end{aligned}
$$

The values given for region 22 are only approximate in the vicinity of region $1(X=0)$ : more precise expressions should be used for the drag number (like Eqs. (58) and (59)).

\section{References}

[1] Ajdari A., Brochard-Wyart F., Gay C., de Gennes P.-G. and Viory J.-L., J. Phys. II France 5 (1995) 491-495.

[2] de Gennes P.-G., C. R. Acad. Scı. 228B (1979) 219.

[3] Brochard-Wyart F. and de Gennes P.-G., Langmurr 8 (1992) 3033-3037.

[4] Brochard-Wyart F., Gay C. and de Gennes P.-G., Macromolecules 29 (1996) 377-382.

[5] de Gennes P.-G., Scaling Concepts in Polymer Physics (Cornell University Press, Ithaca, NY, 4ed., 1985) pp. 219-241.

[6] Graessley W.W., Adv. Polym. Sci.16 (1974).

[7] Brochard-Wyart F., de Gennes P.-G. and Pincus P., C. R. Acad. Sci. 314 II (1992) 873-878.

[8] Assumption was made that only chain $P$ could slide in order to disentangle from grafted chain $N$. In principle, any of the two chains could slide out, but due to grafting, this simple movement is forbidden to chain $N$. The free end of chain $N$ is a rapidly-relaxing part of the chain, and entanglements in this region could dissolve due to grafted chain retraction more rapidly than through the sliding of melt chain $P$ over its whole length. However, this process should dominate only in a small part of the grafted chain near its free end, since the relaxation time is believed to increase exponentially with distance to the free end (see Rubinstein M., Ajdari A., Leibler L., Brochard-Wyart F., de Gennes P.-G., C. R. Acad. Sci. 316 II (1993) 317-320). Hence, the major part of entanglements should be 
renewed through the sliding of the melt chains, as described in the main text, and this free-end effect should not affect the results.

[9] Ajdari A., Brochard-Wyart F., de Gennes P.-G., Leibler L., Viovy J.-L. and Rubinstein M., Physica A 204 (1994) 17-39.

[10] Migler K.B., Hervet H. and Léger L., Phys. Rev. Lett. 70 (1993) 287-290; Migler K.B., Massey G., Hervet H. and Léger L., J. Phys. Condens. Matter 6 (1994) A301-A304; Léger L., Hervet H. and Massey G., ACS Symposium "Interfaces and Surfaces in the Rheology of Polymers" (Anaheim, California, April 1994); Léger L., Hervet H., Marciano Y., Deruelle M. and Massey G., Israel J. Chem. 35 (1995) 65-74.

[11] Massey G., Léger L. and Hervet H., private communication.

[12] Schaefer D.W., Joanny J.-F. and Pincus P., Macromolecules 13 (1980) 1280-1289.

[13] Pincus P., Macromolecules 9 (1976) 386-388.

[14] Marciano Y. and Brochard-Wyart F., Macromolecules 28 (1995) 985-990.

[15] Aubouy M., Fredrickson G.H., Pincus P. and Raphaël E., Macromolecules 28 (1995) 29792981.

[16] Léger L. and Viovy J.-L., Contemp. Phys. 29 (1988) 579-595.

[17] Flory P., Statistics of Chain Molecules (Interscience Publishers, New York, 1969).

[18] Graessley W.W. and Edwards S.F., Polymer 22 (1981) 1329-1334.

[19] Lin Y.-H., Macromolecules 20 (1987) 3080-3083.

[20] Kavassalis T.A. and Noolandi J., Macromolecules 22 (1989) 2709-2720.

[21] For a summary of [19] and [20] in view of [18], see Colby R.H., Rubinstein M. and Viovy J.-L., Macromolecules 25 (1992) 996-998.

[22] Aharony S.M., Macromolecules 16 (1983) 1722-1728.

[23] Raphaël E., Pincus P. and Fredrickson G.H., Macromolecules 26 (1993) 1996-2006; Gay C. and Raphaël E., submitted to J. Phys. II France (Short Commun.). 